\title{
Research on the Organizational Characteristics of Good High School Students' Mathematical Cognitive Structure Based on the Network Block-Modeling Analysis
}

\author{
Sun Dandan ${ }^{1} \&$ Yang Zezhong ${ }^{1}$ \\ ${ }^{1}$ The School of Mathematics\&Statistics, Shandong Normal University, Jinan, China \\ Correspondence: Sun Dandan, The School of Mathematics\&Statistics, Shandong Normal University, Jinan, China. \\ Tel: 86-187-6617-3312. E-mail: 18766173312@163.com
}

Received: March 25, 2017

Accepted: March 31, 2017

Online Published: April 16, 2017

doi:10.5539/jedp.v7n2p14

URL: http://doi.org/10.5539/jedp.v7n2p14

\begin{abstract}
Through the direct detection and quantitative analysis of 44 concepts related to trigonometric functions in the mathematical cognitive structure of the 213 students in grade one in senior middle school, this paper finds that the mathematical cognitive structure of senior high school students has the following characteristics: In the cognitive structure of the mind, knowledge can be divided into different blocks according to the degree of relevance, the basis and the scale of the blocks, the degree of interaction in the block and between the blocks are not the same. The knowledge in a good cognitive structure should be organized in the form of blocks, and the blocks have a more scientific basis. The members in block have more obvious common features and each block is relatively large and covers more knowledge points. The block is closely linked, in addition, there must be a higher intensity of the link between the blocks, which can make the entire network of knowledge as a whole, and then it is conducive to the flow of information.
\end{abstract}

Keywords: mathematical cognitive structure, network analysis, organizational characteristics

\section{Introduction}

Mathematical cognitive structure is a internal structure with distinct individual characteristics, which is created by individuals and based on the studied mathematics knowledge combined with their own cognitive characteristics and non-intelligence factors and so on (Cao C. \& Cai J., 1989; Li, 2001). Mathematical cognitive structure plays an important role in individual's mathematical activities. It not only affects the individual to understand and master the mathematical knowledge, but also affects the individual's application of mathematical knowledge, etc. (Yang, 1993; Yu, 2004). Therefore, in the actual teaching, the teachers expect to help the students to form a good cognitive structure of mathematics. To achieve this goal, there have been many researches on the characteristics of good mathematical cognitive structure in recent years, and achieved fruitful results.

Guan and He believed good mathematical cognitive structure should contain abundant basic knowledge of mathematics, especially a lot of knowledge about the production and the problem solving strategy (Guan, 1998; $\mathrm{He}, 2002)$. Wang, Han and Wang proposed that the characterization of ideas in good mathematical cognitive structure should be reasonable, clear, individualistic, interconnected, and flexible (Wang G. \& Wang Y., 2004; Han \& Wang, 2005). Yu thought that mathematical knowledge in good mathematical cognitive structure may organize together based on the knowledge field and knowledge system. The so-called knowledge field was a knowledge network, in which a large number of knowledge connected with each other according to the equivalence relations, the so-called knowledge system was a series of mathematical knowledge that was formed though abstract or deduction of some knowledge (Yu, 2011).

Review these studies, however, the vast majority are based on the thought of teaching activity experience combined with theory, there is little direct detection and quantitative research of students' mathematical cognitive structure. And, most of these studies concentrate on what content should the good mathematical cognitive structure has, little study is about how to organize the knowledge (Sun \& Yang, 2015; Sun \& Yang, 2015). The research about what content should be contained in good mathematical cognitive structure is 
absolutely important, adopting the method of thinking is also very necessary, but, direct detection and quantitative research of good mathematical cognitive structure, especially of organizational form of knowledge in it is more important, that not only can make the study of good mathematical cognitive structure more in-depth, more clear, but also can make the training of good mathematical cognitive structure more targeted and directional in mathematics teaching, so the author choose part of senior grade one students and do a direct quantitative analysis of characteristics of their mathematical cognitive structure.

\section{Methodology}

\subsection{Participants}

We choose 213 students in grade one in senior middle school, who respectively come from two high schools in Jinan and two in Zibo of Shandong province as the research object. About the selection of top students, this study is based on the current international popular standard, namely the characteristics of usual mathematics learning and the score. The study of mathematical top students is mainly positive, efficient, and their method of study is reasonable, the performance is good and stable.

\subsection{Methods}

By applying the method of concept map and block-modeling, we conduct this study. The method of concept map is used to study the internal cognitive structure based on the concept diagram that individual drew, this method is commonly used to detect individual cognitive structure directly in psychology (Zhang \& Chen, 2000). Block-modeling is one kind of the network analysis method, network analysis is just emerging in recent years, it is a kind of method that is used to analyze network based on the arithmetic of data relation (Liu, 2009). This method is widely used in studies about social network analysis early, and mainly used to study the density, centrality, relationship between subgroups etc. of the network to find out the characteristics of the network and the differences between different networks. Recently, this method is gradually being introduced to the network analysis related to the education and psychology (Luo, 2010). Block modeling is a kind of method used to study network location model, now also has been used in the study of organizational issues, and a large number of small group research, etc.

\subsection{Instrument}

We choose 44 mathematical concepts as study material, which come from the chapter named trigonometric function in high school mathematics textbook compulsory four published by people's education press (44 concepts are as shown in Table 1). We choose this part, because the characteristics of concepts in this part are obvious, the connections between concepts are numerous, and this part is also relatively concentrated in the present high school mathematics teaching.

Table 1. Concepts about trigonometric function

\begin{tabular}{|c|c|c|c|c|c|c|c|}
\hline No. & concept & No. & concept & No. & concept & No. & concept \\
\hline 1 & cyclic transformation & 12 & central angle & 23 & sinusoid & 34 & even function \\
\hline 2 & acute angle & 13 & radian & 24 & phase & 35 & odd function \\
\hline 3 & trigonometric function value & 14 & angle system & 25 & initial phase & 36 & minimal positive period \\
\hline 4 & trigonometric function line & 15 & trigonometric function & 26 & cosine & 37 & period \\
\hline 5 & tangent line & 16 & round angle & 27 & frequency & 38 & periodic function \\
\hline 6 & cosine line & 17 & quadrant angle & 28 & amplitude & 39 & functional images \\
\hline 7 & sine line & 18 & terminal side & 29 & domain of definition & 40 & induction formula \\
\hline 8 & directed line segment & 19 & initial line & 30 & range & 41 & tangent function value \\
\hline 9 & tangent function & 20 & zero angle & 31 & interval & 42 & cosine function value \\
\hline 10 & cosine function & 21 & radian measure & 32 & decreasing function & 43 & obtuse angle \\
\hline 11 & sine function & 22 & arbitrary angles. & 33 & increasing function & 44 & unit circle \\
\hline
\end{tabular}




\subsection{Data Collection}

First, let students draw the map of the relationships between these concepts on paper-that is, the concept map, and mark the compact degree of relationship that they think in the attachment between the adjacent two concepts. The compact degree of relationship is respectively represented with an integer between 1 to 5 from low to high. Then transform the concept map that students draw into one-mode multi-valued relationship matrix in prepare for the analysis of network.

\subsection{Data Analysis}

First, use the network analysis software-Ucinet6.0 and NetDraw to deal with the multi-valued relationship matrix, then compare the situations of top students, medium and general students.

\section{Process}

This study is carried out when the high school students have learned the chapter named trigonometric function in compulsory four. In order to collect the data effectively and easily, the researchers take the way of cluster sampling. In the entire process, the experimenters is always acted by the researchers and mathematics teacher. The process of data collection is as follows: first of all, let the students recall the content in the chapter of trigonometric function, and then present the 44 math concepts that just studied to the students, in order to help students have a more clear understanding of these concepts. Later, let the students draw the map of relationships between these concepts according to their own understanding.

There are 213 students in the four classes in grade one in two senior middle schools, including 96 boys and 117 girls, 31 top students, 127 medium students, and 55 general students. This study finally receive 213 concept maps, 7 sets are rejected because they don't meet the requirements (mainly because the picture is not clear and disorderly), we get 206 concept maps that are suitable for further analysis. For the 206 concept maps, the researchers transform them into a one-mode multi-valued relationship matrix first, and carry on the preliminary sorting depending on the type of the level of the students, then analyze the block modeling of concept networks using network software Ucinet6.0. The materials of middle and general students are also surveyed and analyzed here, the reason is that we want put their data as compare objects, so that we can analyze the characteristics of top students' mathematical cognitive structure more in-depth.

\section{Results}

In order to analysis the structure of knowledge network, for different types of knowledge networks, at first, we calculate the number of subgroups (locations) in the network and then show density and image matrix between different positions, at last show the simplified diagram of the relationship. It can be clearly seen that the knowledge network is divided into a number of "gangs" and how they are connected with each other. For this purpose, the three kinds of mean value matrix of the top students, the medium students and the general students are treated symmetrically, and the tree diagrams of the network are divided into 8 blocks by the Concor method.

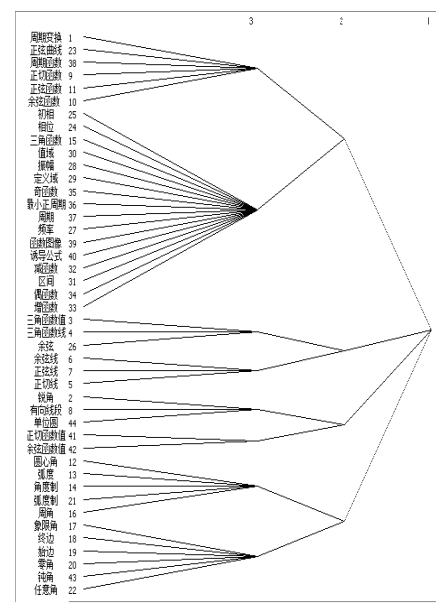

Figure 1. Top students 


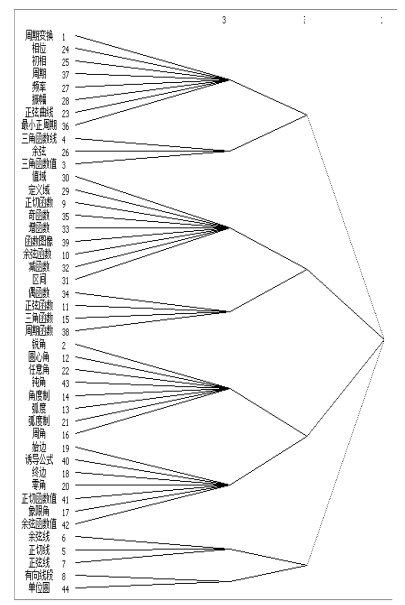

Figure 2. Medium students

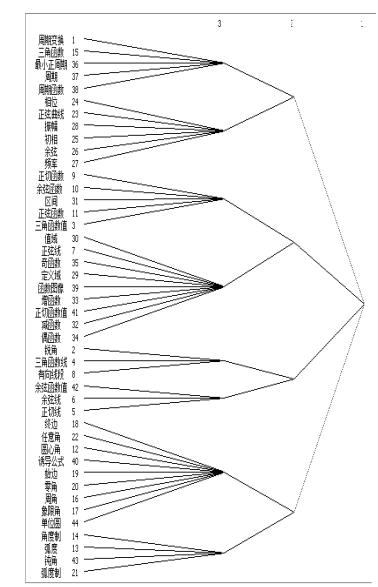

Figure 3. General students

It can be seen from the above three diagrams, whether it is condensed into four subgroups or eight subgroups, the above 44 mathematical concepts can be divided into a large subgroup and a few subgroups in the cognitive structure of the top students, instead, in the cognitive structure of medium students and the general students, they are basically the small subgroups with relatively average number of elements, without large subgroup with obvious numerous elements. This situation is more obvious with the increasing of cohesive subgroups.

When condensed into eight subgroups, the condensation condition of the above 44 mathematical concepts in the top students', medium students' and the general students' cognitive structure is very different. The common features of the majority of subgroups in the cognitive structure of top students are more obvious, and the relationship between each other is more clear and reasonable. For example, the concepts of the top students' second subgroup are basically about the basic properties of trigonometric functions. The concepts of the fourth subgroup of the top students is closely related to the trigonometric function line. The concepts of the sixth subgroup are obviously related to the trigonometric function value. The concepts of the seventh subgroup are related to the radian system. The concepts of the eighth subgroup are related to arbitrary angle. Moreover, when the whole network is divided into 4 blocks, the first and the second subgroups of the top students will condense into a group further which contain all the key concepts of the basic properties of trigonometric functions. In detail, the third and the fourth subgroups can be regarded as the expansion of trigonometric function line; the seventh and the eighth subgroups are all based on the comparative basis of arbitrary angles and radians. Using the same analytical thinking to look backward the subgroup of medium students and general students, we can find some general and common features. For example, the concepts of the first subgroup of medium students are related to the properties of trigonometric functions; the concepts of the seventh and the eighth subgroups are 
related to the trigonometric function line; the concepts of the second subgroup of general students are related to the properties of trigonometric functions. However, from the perspective of each subgroup's members of medium students and general students, it's not very easy to find common points because many subgroups are associated with other uncorrelated concepts. For example, the seventh subgroup of the general students is almost about arbitrary angle, but also doping induction formula and unit circle. Secondly, from a macro point of view, there is a very obvious and important difference among the top, medium and general students. The property of the trigonometric function in this chapter is a very important knowledge point. In the cognitive structure of top students, the unique properties of trigonometric functions such as initial phase, phase and amplitude can be combined with the properties of the functions previously studied, such as monotonicity and parity and then form an organic whole and reflected in the second subgroup. However, these two parts of properties in the cognitive structure of medium students and general students scatter in three subgroups. Such knowledge organization is obviously not so scientific as that of the top students which link the previous and following knowledge closely according to a certain standard of knowledge. It is worth noting that in the third subgroup of medium students, although it is mainly about general function there are two kinds of trigonometric functions, which shows that there is intention to contact the two parts' knowledge. However, this kind of connection is weaker in the subgroups of general students. At this time, the density matrix of top students, medium students and general students is shown in Tables 2, 3, 4 .

Table 2. The density matrix of top students

\begin{tabular}{lllllllll}
\hline No. & 1 & 2 & 3 & 4 & 5 & 6 & 7 & 8 \\
\hline 1 & 1.11 & 1.12 & 0.46 & 0.46 & 0.07 & 0.20 & 0.04 & 0.01 \\
2 & 1.12 & 0.31 & 0.10 & 0.24 & 0.05 & 0.22 & 0.03 & 0.02 \\
3 & 0.46 & 0.10 & 0.85 & 1.70 & 0.49 & 0.15 & 0.03 & 0.08 \\
4 & 0.46 & 0.24 & 1.70 & 0.72 & 1.23 & 0.27 & 0.00 & 0.03 \\
5 & 0.07 & 0.05 & 0.49 & 1.23 & 0.23 & 0.00 & 0.26 & 0.41 \\
6 & 0.20 & 0.22 & 0.15 & 0.27 & 0.00 & 0.75 & 0.11 & 0.38 \\
7 & 0.04 & 0.03 & 0.03 & 0.00 & 0.26 & 0.11 & 1.21 & 0.33 \\
8 & 0.01 & 0.02 & 0.08 & 0.03 & 0.41 & 0.38 & 0.33 & 1.11 \\
\hline
\end{tabular}

Table 3. The density matrix of medium students

\begin{tabular}{lllllllll}
\hline No. & 1 & 2 & 3 & 4 & 5 & 6 & 7 & 8 \\
\hline 1 & 0.36 & 0.21 & 0.12 & 0.26 & 0.02 & 0.01 & 0.06 & 0.01 \\
2 & 0.21 & 0.73 & 0.15 & 0.19 & 0.05 & 0.09 & 0.54 & 0.08 \\
3 & 0.12 & 0.15 & 0.67 & 0.48 & 0.01 & 0.05 & 0.04 & 0.00 \\
4 & 0.26 & 0.19 & 0.48 & 0.46 & 0.07 & 0.10 & 0.07 & 0.08 \\
5 & 0.02 & 0.05 & 0.01 & 0.07 & 0.42 & 0.14 & 0.02 & 0.15 \\
6 & 0.01 & 0.09 & 0.05 & 0.10 & 0.14 & 0.34 & 0.03 & 0.09 \\
7 & 0.06 & 0.54 & 0.04 & 0.07 & 0.02 & 0.03 & 0.64 & 0.67 \\
8 & 0.01 & 0.08 & 0.00 & 0.08 & 0.15 & 0.09 & 0.67 & 0.00 \\
\hline
\end{tabular}


Table 4. The density matrix of general students

\begin{tabular}{lllllllll}
\hline No. & 1 & 2 & 3 & 4 & 5 & 6 & 7 & 8 \\
\hline 1 & 0.65 & 0.23 & 0.21 & 0.08 & 0.01 & 0.03 & 0.02 & 0.00 \\
2 & 0.23 & 0.24 & 0.09 & 0.02 & 0.00 & 0.03 & 0.00 & 0.00 \\
3 & 0.21 & 0.09 & 0.06 & 0.36 & 0.07 & 0.20 & 0.04 & 0.00 \\
4 & 0.08 & 0.02 & 0.36 & 0.10 & 0.06 & 0.06 & 0.02 & 0.00 \\
5 & 0.01 & 0.00 & 0.07 & 0.06 & 0.17 & 0.44 & 0.03 & 0.05 \\
6 & 0.03 & 0.03 & 0.20 & 0.06 & 0.44 & 0.14 & 0.08 & 0.00 \\
7 & 0.02 & 0.00 & 0.04 & 0.02 & 0.03 & 0.08 & 0.30 & 0.12 \\
8 & 0.00 & 0.00 & 0.00 & 0.00 & 0.05 & 0.00 & 0.12 & 0.43 \\
\hline
\end{tabular}

The density matrix is a symmetric matrix and the number in the diagonal position represents the density of each subgroup, the number in the remaining position represents the correlation density of corresponding rows and columns of subgroups. It can be seen from Tables 2, 3, 4, the density of the cohesive subgroups of the top students is range from 0 to 1.7 , with an average about 0.36 . The density of the cohesive subgroups of the medium students is range from 0 to 0.728 , with an average about 0.17 . The density of the cohesive subgroups of the general students is range form 0 to 0.65 , with an average about 0.10 . No matter in terms of the maximum density or the average density, the top students are both higher than the medium students, the medium students are higher than the general students. This shows that, on the whole, the degree of connection relationship between different subgroups in the cognitive structure of the top students is generally greater than that of the medium students. On the basis of the relationship in and between the subgroups of the cognitive structure network, the top students are better than the medium students and the medium students are better than the general students.

It can be also seen from Tables 2, 3, 4, that the maximum degree of closeness between the different subgroups in the cognitive structure of the top students is about 1.7- the third subgroup and the fourth subgroup. However, the maximum degree of connection between different subgroups in the cognitive structure of medium students is only 0.667 - the seventh subgroup and the eighth subgroup. The maximum degree of connection between different subgroups in the cognitive structure of general students is 0.444 - the fifth subgroup and the sixth subgroup. Interestingly, the maximum degree of closeness of top students, medium students, general students both appears between two subgroups trigonometric - function line, directed line segment and sine line, cosine line, tangent line, which is also a scientific fact that is in accord with objective conditions.

The densities of the multi valued symmetric matrix networks of the general students, the medium students and the top students are respectively $0.0928,0.1533,0.3279$. Regard the density of three types of network as the critical value. Then, the density of the corresponding density matrix higher than the critical value is recorded as 1 , and the density less than the critical value is recorded as 0 . Finally, we get the image matrix of general students, medium students and top students and the simplified relation diagram can be got according to it.

Image matrix is a two numerical value symmetric matrix. When the numerical value is 1 , it indicates that there is a connection between corresponding block in the sense of higher than average density. When the numerical value is 0 , there is no connections. Each circle in the simplified diagram represents a subgroup, curves with arrows on each circle represents the relationship from the circle "issued" back to the circle, that is, the density of the subgroup is higher than the overall matrix density. If there is a connection between circles, which means in the sense of higher than average density there is a "communication" between subgroups. If there is no connection between points that means "no communication". 
Table 5. Image matrix of top students

\begin{tabular}{lllllllll}
\hline No. & 1 & 2 & 3 & 4 & 5 & 6 & 7 & 8 \\
\hline 1 & 1 & 1 & 1 & 1 & 0 & 0 & 0 & 0 \\
2 & 1 & 0 & 0 & 0 & 0 & 0 & 0 & 0 \\
3 & 1 & 0 & 1 & 1 & 1 & 0 & 0 & 0 \\
4 & 1 & 0 & 1 & 1 & 1 & 0 & 0 & 0 \\
5 & 0 & 0 & 1 & 1 & 0 & 0 & 0 & 1 \\
6 & 0 & 0 & 0 & 0 & 0 & 1 & 0 & 1 \\
7 & 0 & 0 & 0 & 0 & 0 & 0 & 1 & 1 \\
8 & 0 & 0 & 0 & 0 & 1 & 1 & 1 & 1 \\
\hline
\end{tabular}

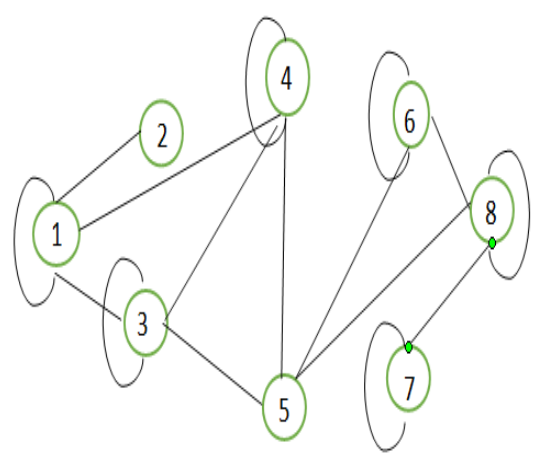

Figure 4. Simplified diagram of top students

Table 6. Image matrix of medium students

\begin{tabular}{lllllllll}
\hline No. & 1 & 2 & 3 & 4 & 5 & 6 & 7 & 8 \\
\hline 1 & 1 & 1 & 0 & 1 & 0 & 0 & 0 & 0 \\
2 & 1 & 1 & 0 & 1 & 0 & 0 & 1 & 0 \\
3 & 0 & 0 & 1 & 1 & 0 & 0 & 0 & 0 \\
4 & 1 & 1 & 1 & 1 & 0 & 0 & 0 & 0 \\
5 & 0 & 0 & 0 & 0 & 1 & 0 & 0 & 0 \\
6 & 0 & 0 & 0 & 0 & 0 & 1 & 0 & 0 \\
7 & 0 & 1 & 0 & 0 & 0 & 0 & 1 & 1 \\
8 & 0 & 0 & 0 & 0 & 0 & 0 & 1 & 0 \\
\hline
\end{tabular}




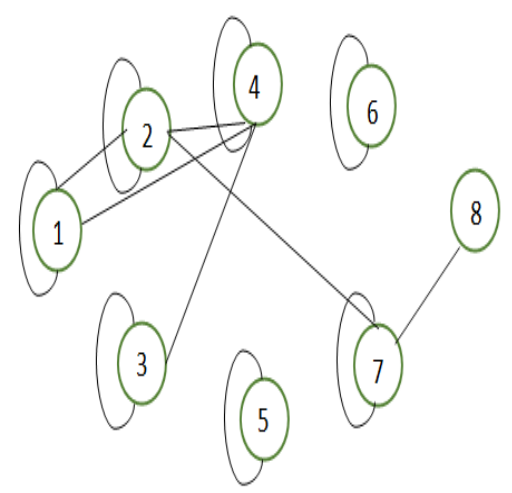

Figure 5. Simplified diagram of medium students

Table 7. Image matrix of medium students

\begin{tabular}{ccccccccc}
\hline $\mathrm{N}$ & 1 & 2 & 3 & 4 & 5 & 6 & 7 & 8 \\
\hline 1 & 1 & 1 & 1 & 0 & 0 & 0 & 0 & 0 \\
2 & 1 & 1 & 0 & 0 & 0 & 0 & 0 & 0 \\
3 & 1 & 0 & 0 & 1 & 0 & 1 & 0 & 0 \\
4 & 0 & 0 & 1 & 1 & 0 & 0 & 0 & 0 \\
5 & 0 & 0 & 0 & 0 & 1 & 1 & 0 & 0 \\
6 & 0 & 0 & 1 & 0 & 1 & 1 & 0 & 0 \\
7 & 0 & 0 & 0 & 0 & 0 & 0 & 1 & 1 \\
8 & 0 & 0 & 0 & 0 & 0 & 0 & 1 & 1 \\
\hline
\end{tabular}

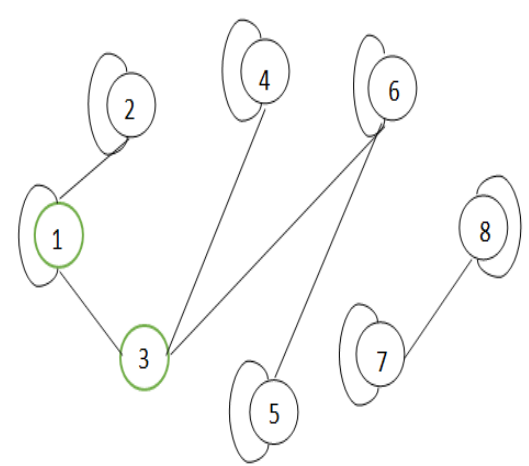

Figure 6. Simplified diagram of general students

It can be seen clearly that there are no scattered subgroups in the concept network of top students. In details, there is a close connection between the 8 positions. The fifth and the sixth subgroups of medium students are isolated and the seventh and the eighth subgroups of general students are not associated with other subgroups. The relatively isolated subgroups of the concept network of medium and general students are arbitrary angles and radians. In addition, there is mutual relation in the simplified relationship diagram of top students and medium students. In this case, three small groups may form a relatively large group which is conducive to the circulation of information, whereas a similar situation does not appear in the simplified concept diagrams of general students. The relationship between the subgroups of general students is basically linear, and there is no greater subgroup. 


\section{Conclusions and Suggestion}

In the cognitive structure of the mind, knowledge can be divided into different blocks according to the degree of relevance. The basis and the scale of the blocks, the degree of interaction in the block and between the blocks are not the same. In the knowledge structure of the top students, there are often a large block and a few small blocks when the knowledge is divided, nevertheless the members of the blocks of the medium students and the general students have relatively little difference. In details, the top students may embeds the specific knowledge in the higher level of knowledge, thus, a more general relation between knowledge is established, and a large knowledge block is obtained. The classification of the blocks of top students is more scientific, and the commonness of the members in the blocks is more clearer. In addition, the density in the block and between the blocks is relatively large, that is, the connection of the knowledge in the block and between the blocks is the most compact. The knowledge in a good cognitive structure should be organized in the form of blocks, and the blocks have a more scientific basis. The members in block have more obvious common features and each block is relatively large and covers more knowledge points. The block is closely linked, in addition, there must be a higher intensity of the link between the blocks, which can make the entire network of knowledge as a whole, and then it is conducive to the flow of information.

Based on this, in the teaching process, first of all, teachers should guide students to build knowledge blocks, that is, to teach clearly about the relationship between knowledge so that the knowledge scattered in the cognitive structure of students can be made into a block which has great benefit to students' knowledge memory, extraction and operation. In addition, teachers should not only pay attention to guide students to establish a clear knowledge block, but also strive to guide students to expand the scale of knowledge blocks. How to expand it? Starting from the edge knowledge points of the established knowledge blocks, we can establish the further connection between these knowledge points and other concepts, so as to expand the scale of knowledge blocks.

\section{Acknowledgments}

Supported by the project of research on organizational characteristic of mathematical cognitive based on the network analysis (SCX201614).

\section{References}

Cao, C. H., \& Cai, J. F. (1989). Introduction to Mathematics Education (pp. 52-53). Nanjing: Jiangsu Education Press.

Guan, P. (1998). Principles of Cognitive Psychology on Forming Good Mathematical Cognitive Structure. Theory and Practice of Education, 18(2), 40-45.

Han, B., \& Wang, G. M. (2005). Thinking about the Role of Cognitive Structure in the Process of Problem Solving. Journal of Middle School Mathematics (High School), 6, 5-7.

He, X. Y. (2002). Instructional Strategies on Constructing well Mathematical Cognitive Structure. Journal of Mathematics Education, 11(1), 24-27.

Li, S. Q. (2001). PME: Psychology of Mathematics Education (pp. 24-25). Shanghai: East China Normal University Press.

Liu, J. (2009). Lectures on Whole Network Approach (pp. 17-38). Shanghai: Gezhi Press.

Luo, J. D. (2010). Social Network Analysis (pp. 47-57). Beijing: Social Sciences Academic Press.

Sun, D. D., \& Yang, Z. Z. (2015). Research on Good Mathematical Cognitive Structure in Mainland China. Advances in Social and Behavioral Sciences: Proceeding of 2015 3rd Asian Conference on the Social Sciences, 15, 216-219.

Sun, D. D., \& Yang, Z. Z. (2015). Study on Content and Organization of Mathematical Cognitive Structure in Mainland China. Advances in Intelligent Systems Research, 125, 1521-1525. https://doi.org/10.2991/meici-15.2015.283

Wang, G. M., \& Wang, Y. (2004). The comparison of the mathematical cognitive structure between the top and ordinary student in senior high school, the possible reason and teaching suggestions. Preference for Middle School Mathematical Education, 12, 1-4.

Yang, Q. (1993). An Study on The Influence of Cognitive Structure to Mathematics Learning-One of the Explores about the Psychological Factors That Influences the Mathematics Learning. Journal of Mathematics Education, 2(1), 66-70. 
Yu, P. (2004). Influence of Self-controlling Ability and CPFS Structure on the Mathematical Achievement in High School Students. Journal of Mathematics Education, 13(1), 23-26.

Yu, P. (2011). China's Psychology Research of Mathematics Education in 30 Years. Beijing: Science Press.

Zhang, J. W., \& Chen, Q. (2000). Test Method of Cognitive Structure. Journal of Psychological Science, 6, $750-751$.

\section{Copyrights}

Copyright for this article is retained by the author(s), with first publication rights granted to the journal.

This is an open-access article distributed under the terms and conditions of the Creative Commons Attribution license (http://creativecommons.org/licenses/by/4.0/). 